\title{
Downregulation of microRNA-429 protects cardiomyocytes against hypoxia-induced apoptosis by increasing Notch1 expression
}

\author{
HAN XU $^{1}$, LONG JIN ${ }^{2}$, YAN CHEN ${ }^{1}$ and JUNZHI LI ${ }^{1}$ \\ ${ }^{1}$ Department of Emergency and ${ }^{2}$ The Second Department of Geriatrics, \\ The Ninth Hospital of Xi'an City, Xi'an, Shaanxi 710054, P.R. China
}

Received October 29, 2015; Accepted March 17, 2016

DOI: $10.3892 /$ ijmm.2016.2558

\begin{abstract}
Myocardial ischemia is a commonly encountered symptom, chiefly as a result of coronary artery and heart diseases in middle-aged and elderly individuals, with a sudden occurrence and a high morbidity. In some cases, myocardial ischemia may lead to the injury and subsequent death of cardiomyocytes, and may finally culminate in myocardial infarction (MI). MI is the leading cause of sudden death and is associated with a high mortality rate.In this study, we focused on the role of microRNA-429 (miR-429) in protecting the cardiomyocytes against apoptosis induced by myocardial ischemia. The culture of human cardiomyocytes under hypoxic conditions was employed to mimic myocardial ischemia. miR-429 expression was upregulated following culture under hypoxic conditions. Subsequently, miR-429 was artificially overexpressed and silenced by transfection with miRNA-mimics and miRNA-inhibitor, respectively. The results revealed that the downregulation of miR-429 expression exerts protective effects against hypoxia-induced apoptosis. Moreover, Notch1 was also proven to be involved in these protective effects. The downregulation of miR-429 was accompanied by the activation of Notch1, as indicated by the significant increase in the protein expression of Notch1. The ectopic expression of Notch1 also inhibited the apoptosis induced by culture under hypoxic conditions. In conclusion, and to the best of our knowledge, our results demonstrate for the first time that the downregulation of miR-429 protects cardiomyocytes against hypoxia-induced apoptosis through Notch1; this may provide the experimental basis for an underlying therapeutic target for myocardial ischemia and consequent MI, as well as the basis for an effective preventive treatment against sudden death.
\end{abstract}

Correspondence to: Dr Long Jin, The Second Department of Geriatrics, The Ninth Hospital of Xi'an City, 151 Eastern part of South 2nd Ring Road, Xi'an, Shaanxi 710054, P.R. China E-mail: longjindragon@163.com

Key words: microRNA-429, cardiomyocytes, sudden cardiac death, apoptosis, Notch1

\section{Introduction}

Coronary artery and heart diseases are common diseases affecting the elderly, and are often associated with a high morbidity. Myocardial ischemia is the main symptom of coronary artery and heart diseases, which is characterized by a reduction in blood perfusion and oxygen supply, causing heart dysfunction and deficiency (1). Myocardial ischemia is always followed by myocardial injury and the apoptosis of the cardiac muscle cells, eventually leading to myocardial infarction (MI) and finally to the death of patients, commonly termed sudden cardiac death in this case $(2,3)$. Hence, research into myocardial ischemic injury may provide an experimental basis for the exploration of sudden cardiac death. It has been demonstrated that myocardial apoptosis is the most destructive event induced by myocardial ischemia and oxygen reperfusion (4). It is also widely known that apoptosis is one of the main types of programmed cell death (5). Consequently, based on the descriptions given above, we hypothesized that the inhibition of apoptosis may provide a protective effect against myocardial ischemia and possible sudden death. Moreover, an in-depth molecular understanding of the apoptotic pathway would provide a better understanding of the mechanisms behind myocardial ischemia and may provide effective treatments for myocardial ischemic injury, and for the prevention of sudden death.

Hypoxia is closely related to cardiovascular diseases due to the insufficient oxygen supply; in particular, hypoxia is one of the harmful consequences of myocardial ischemia, which triggers a wide range of cellular responses, including gene regulation and cell apoptosis (6). However, hypoxia-induced cell apoptosis is rarely reported in human cardiomyocytes. In the present study, the culture of human cardiomyocytes under hypoxic conditions was employed to mimic cardiac ischemia.

MicroRNAs (miRNAs or miRs) are a class of 17-25 nucleotide, non-coding small RNA molecules, regulating the expression of their target genes at the post-transcriptional level by binding to the 3'-untranslated region (3'-UTR) of the target mRNA and degenerating the target mRNA (7). A variety of miRNAs have been reported to regulate myocardial hypoxiainduced apoptosis (8-10). For example, miR-133a was reported to be downregulated in hypoxia-treated $\mathrm{H} 9 \mathrm{c} 2$ cells, although the overexpression of miR-133a was shown to inhibit hypoxiainduced apoptosis (11). In addition, in hypoxia-exposed neonatal rat cardiomyocytes, miR-26b was shown to be upregulated; 
however, the downregulation of miR-26b exerted inhibitory effects on hypoxia-induced apoptosis (12). miR-429 belongs to the miR-200 family, which is dysregulated in various types of cancer. For example, miR-429 has been reported to play a role in colorectal cancer (14), hepatocellular carcinoma $(13,15)$, esophageal carcinoma (16), breast cancer (17) and oral squamous cell carcinoma (18), and its expression and functions (inhibitory or promotive effects on cancer development) vary with and are dependent upon the type of tumor. Moreover, Gao and Liu reported that in hepatocellular carcinoma cells, miR-429 directly targets Notch 1 and reduces both the mRNA and protein levels of Notch1 (15). However, the precise functions and effects of miR-429 in myocardial ischemia and subsequent apoptosis have not been fully investigated to date.

The Notch pathway participates in various cellular processes, including cell proliferation, differentiation and apoptosis during the embryonic and adult stages $(19,20)$. In particular, the Notch pathway has been reported to be associated with cardiac repair, regeneration and protection (21). In fact, the Notch pathway controls mesodermal differentiation during embryonic and adult development, including cardiac differentiation $(22,23)$. Moreover, the activation of the Notch pathway has been shown to exert inhibitory effects on the degree of ischemic injury and to improve heart function following MI (24). The Notch receptors are important effectors of the Notch pathway, which regulates cardiovascular development and homeostasis $(25,26)$. In mammals, 4 Notch receptors (Notch1-4) and 5 Notch ligands have been identified (27), among which Notch1 is strongly expressed in cardiomyocytes, smooth muscle cells and endothelial cells (21). Notch1 has been reported to play a role in promoting the proliferation of immature cardiomyocytes in rat models, and the inhibition of the Notch pathway inhibits proliferation and induces apoptosis (28). Yu and Song demonstrated that Notch1 reduced the apoptosis of rat cardiomyocytes in ischemic post-conditioning, and the activation of the Notch1 gene markedly promoted cell proliferation and inhibited cell apoptosis (29). However, although the results mentioned above demonstrate the protective and promoting effects of Notch1 in cardiac development, very little is known regarding the underlying mechanisms of the function of Notch1 in this area. In addition, the interactions between miRNAs and Notch1 are not yet fully understood, particularly in human cardiomyocytes. We hypothesized that Notch1 may play an important role in alleviating the hypoxia-induced apoptosis of human cardiomyocytes and, therefore, we designed the subsequent experiments to verify our hypothesis.

In this study, the culture of human cardiomyocytes under hypoxic conditions was employed to mimic the insufficient oxygen supply induced by myocardial ischemia. The expression of miR-429 was evaluated, and it was artificially overexpressed and silenced under hypoxic conditions. Subsequently, the effects of miR-429 on cell apoptosis were investigated. Notch1 was introduced into this study as it was identified as one of the target genes of miR-429 in the homo sapiens species. Moreover, the effects of Notch1 expression on cell apoptosis were further explored. In sum, our results provide new insight into cell apoptosis induced by myocardial ischemia and the cause of sudden death, which may lead towards the discovery of novel therapeutic methods targeting Notch1 and to the more effective prevention from myocardial ischemia and ensuing sudden death.

\section{Materials and methods}

Cell culture and exposure to hypoxia. Human cardiomyocytes (AC-16), purchased from the Cell Resource Center of Shanghai Institutes for Biological Sciences, Chinese Academy of Sciences, were cultured in RPMI-1640 medium supplemented with $10 \%$ fetal bovine serum (FBS) (both from HyClone, Logan, UT, USA) in an atmosphere of $37^{\circ} \mathrm{C}$ with $5 \% \mathrm{CO}_{2}$. For culture under hypoxic conditions, the cells were incubated in a hypoxic $\left(1 \% \mathrm{O}_{2} / 5 \% \mathrm{CO}_{2} / 94 \% \mathrm{~N}_{2}\right)$ atmosphere at $37^{\circ} \mathrm{C}$ for $12 \mathrm{~h}$. The cells cultured under normoxic conditions $\left(5 \% \mathrm{CO}_{2} / 95 \%\right.$ air) served as the controls.

Transfection with miRNA-mimics and miRNA-inhibitor. To induce miR-429 overexpression or silencing, the cells were transfected with negative control miRNA (NC miRNA), miRNA-mimics and miRNA-inhibitor (GenePharma Co., Ltd., Shanghai, China) using Lipofectamine 2000 (Invitrogen, Carlsbad, CA, USA) following the manufacturer's insructions.

The ectopic expression of Notchl. Adenoviral vectors were constructed by GenePharma Co., Ltd. Briefly, the adenoviruses were amplified and titrated in 293 cells. The AC- 16 cells were transiently transfected with adenoviruses carrying the Notch1 gene (Ad-Notch1) without any obvious signs of cytotoxicity [determined by the fact that the transfected AC- 16 cells survived as usual without sudden death, and the morphology of transfected AC-16 cells was also unaltered (data not shown)] using Lipofectamine 2000 (Invitrogen) following the manufacturer's instructions. Some of the AC-16 cells were transfected with the empty carrier LacZ (Ad-LacZ) as the negative control (NC) group. For the co-overexpression of miR-429 and Notch1, the cells were co-transfected with miRNA-mimics and Ad-Notch1 harboring no specific binding sequences of miR-429 in the 3'-UTR using Lipofectamine 2000 (Invitrogen) according to the manufacturer's instructions.

3-(4,5-Dimethylthiazol-2-yl)-2,5-diphenyltetrazolium bromide (MTT) assay. Cell viability was evaluated using the MTT Cell Proliferation and Cytotoxicity assay kit (Beyotime, Jiangsu, China). Briefly, the cells were seeded into 96-well dishes at a density of $1 \times 10^{4}$ cells/well, and were then subjected to hypoxic conditions as described above. Subsequently, $20 \mu 1$ of MTT solution $(5 \mathrm{mg} / \mathrm{ml})$ were added to each well followed by incubation for a further $4 \mathrm{~h}$. The medium was then discarded and $150 \mu \mathrm{l}$ of dimethylsulfoxide (DMSO; Sigma-Aldrich, St. Louis, MO, USA) was added with rotation for $10 \mathrm{~min}$ to dissolve the formazan. Finally, the optical density (OD) value of each well at $490 \mathrm{~nm}$ was measured and recorded using a microplate spectrophotometer (NanoDrop Technology, Wilmington, DE, USA); this was set as the Y-axis for the viability curve. Each well was measured in triplicate.

5-Bromo-2-deoxyuridine (BrdU) assay. Cell proliferation was assessed by BrdU assay using the BrdU Labeling and Detection kit II (Roche, Basel, Switzerland) following the manufacturer's instructions. Briefly, the cells were seeded in 96-well dishes at a density of $1 \times 10^{4}$ cells/well, and were then subjected to indicated treatments/conditions. Subsequently, BrdU labeling solution was added to each well at a final concentration of $10 \mu \mathrm{M}$ followed 
by a further $4 \mathrm{~h}$ of incubation at $37^{\circ} \mathrm{C}$. The cells were then fixed with $4 \%$ cold paraformaldehyde for $20 \mathrm{~min}$ and washed thrice with phosphate-buffered saline (PBS), followed by incubation in $1.5 \mathrm{M} \mathrm{HCl}$ for $30 \mathrm{~min}$ for denaturalization. The cells were then incubated with peroxidase-labeled anti-BrdU antibody (1:100, provided with the kit) at room temperature. The cells were counterstained with hematoxylin, and the BrdU-positive cells and total cells were counted in 5 randomly selected fields at x400 magnification. Cell proliferation was demonstrated as the labeling index (LI) of BrdU-positive cells vs. total cells.

Terminal deoxynucleotide transferase-mediated nick endlabeling (TUNEL) assay. The cells were seeded in 6-well dishes at a density of $1 \times 10^{5}$ cells/well, followed by exposure to hypoxic conditions and transfection as described above. TUNEL assay was used to detect the apoptotic cardiomyocytes following myocardial ischemia. Briefly, the cells were fixed with 4\% paraformaldehyde for $30 \mathrm{~min}$, washed and incubated on ice with $0.1 \%$ Triton X-100 (Sigma-Aldrich) for $2 \mathrm{~min}$. TUNEL staining was performed using the TUNEL detection kit (Roche) according to the manufacturer's instructions. Finally, the cells were counterstained with hematoxylin after TUNEL staining. Five microscopic fields in each well were randomly selected to count the TUNEL-positive cells and the total cells at $\mathrm{x} 400$ magnification. The ratio of the TUNEL-positive cells vs. the total number of cells was calculated as the apoptotic rate.

Hoechst staining. The cells were digested and subcultured into 96-well plates at a density of $5 \times 10^{3}$ cells/well. Subsequently, the cells were subjected to transfection as described above. Cell apoptosis was detected using the Hoechst staining kit(Beyotime) by counting the number of stained cell nuclei according to the manufacturer's instructions. The positively stained cells were visualized under a fluorescence microscope (Olympus, Tokyo, Japan) and counted in randomly selected fields for calculating the apoptotic rate.

Detection of caspase- 3 activity. The supernatant of the treated cells was collected and examined using the colorimetric caspase-3 assay kit (Beyotime) to detect the caspase-3 activity according to the manufacturer's instructions. Briefly, the AC-16 cells were lysed on ice, and $5 \mathrm{mg}$ of the caspase- 3 substrate were then added to the lysates, and the samples were incubated for $4 \mathrm{~h}$ at $37^{\circ} \mathrm{C}$. The absorbance at $405 \mathrm{~nm}$ was measured using a microplate spectrophotometer (NanoDrop Technology).

Target prediction. The target genes of miR-429 were predicted from 3 established miRNA-target prediction programs: TargetScan (http://www.targetscan.org/), Pictar (http://pictar. mdc-berlin.de/cgi-bin/new_PicTar_mouse.cgi) and microRNA. org (http://www.microrna.org/microrna/home.do) at the same time.

Dual-luciferase reporter assay. The mutant type (Mut) 3'-UTR was constructed by replacing certain miR-429 binding sites in the 3'-UTR of the Notch1 with other bases. The Notch1 wild-type (WT) 3'-UTR and Mut 3'-UTR were amplified, and subsequently transfected into a luciferase reporter vector (pmiR-REPORT; Ambion, Rockville, MD, USA) to generate the recombinant plasmids of the pmiR-Notch1-WT and pmiR-

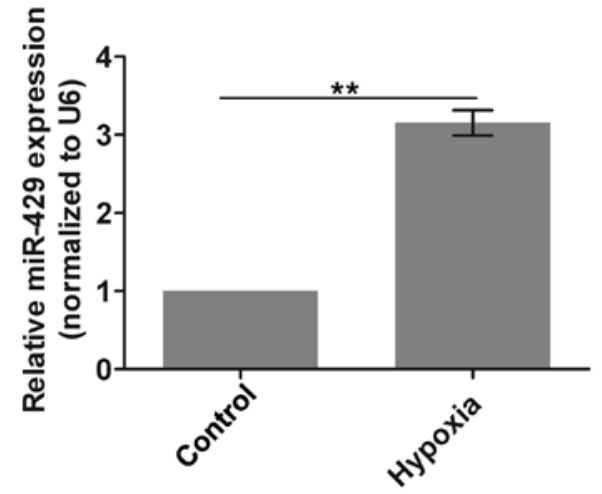

Figure 1. miR-429 expression was increased following culture under hypoxic conditions. Compared with the control group cultured under normoxic conditions, the miR-429 was significantly upregulated by exposure to hypoxia. Each experiment was performed in triplicate; ${ }^{* *} \mathrm{P}<0.01$ in comparison with the control group.

Notch1-Mut. The NC miRNA and miRNA-mimics were co-transfected with the pmiR-Notch1-WT and pmiR-Notch1Mut, mutually, into the 293 cells using Lipofectamine 2000 reagent (Invitrogen) according to the manufacturer's instructions. The luciferase activity was examined $24 \mathrm{~h}$ later using the Dual-Luciferase Reporter assay system (Promega, Madison, WI, USA).

Reverse transcription-quantitative PCR (RT-qPCR). Total RNA was extracted from the AC-16 cells usingTRIzol ${ }^{\circledR}$ reagent (Invitrogen) and then reverse-transcribed into cDNA using the RevertAid First Strand cDNA Synthesis kit (Thermo Fisher Scientific, Waltham, MA, USA). qPCR was performed using SYBR ${ }^{\circledR}$ Fast qPCR Mix (Takara Biotechnology, Dalian, China) on the CFX96 Real-Time PCR Detection System (Bio-Rad, Hercules, CA, USA). qPCR was then conducted under the following conditions: $95^{\circ} \mathrm{C}$ for $10 \mathrm{~min}, 40$ cycles at $95^{\circ} \mathrm{C}$ for $2 \mathrm{~min}, 60^{\circ} \mathrm{C}$ for $12 \mathrm{sec}$, and $72^{\circ} \mathrm{C}$ for $30 \mathrm{sec}$. U6 served as the internal reference gene for miRNAs and glyceraldehyde 3-phosphate dehydrogenase (GAPDH) for the other genes. The relative expression level was normalized to that of the internal reference gene using the $2^{-\Delta \Delta \mathrm{Ct}}$ cycle threshold method.

Western blot analysis. Total protein was extracted using RIPA buffer (Sigma-Aldrich) as follows: the medium was discarded and RIPA buffer was added, with rotation for $10 \mathrm{~min}$ on ice for protein lysis. The protein lysates were collected using a scraper and separated by $10 \%$ sodium dodecyl sulfonate-polyacrylamide gel electrophoresis (SDS-PAGE) and then electroblotted onto the PVDF membranes (Millipore, Billerica, MA, USA), which were subsequently incubated with the primary antibodies, including rabbit anti-caspase-3 (ab408; 1:2,000), goat antiBcl-2 (ab37899; 1:2,000), rabbit anti-Bax (ab104156; 1:1,500) and rabbit anti-GAPDH (ab57062; 1:2,000) overnight at $4^{\circ} \mathrm{C}$, followed by incubation with secondary antibodies (ab97196 and ab6880; 1:4,000) (all from Abcam, Cambridge, UK) at $37^{\circ} \mathrm{C}$ for $4 \mathrm{~h}$. An enhanced chemiluminescence detection system was used for visualizing the bound proteins. Densitometric analysis was performed using Image-Pro Plus (Roper Industries, New York, NY, USA), and the densitometry of the immunoblots was normalized to GAPDH. The densitometry of the most abundant 
band in each experiment was set as $100 \%$, and other bands were normalized to $100 \%$.

Statistical analysis. All data are presented as the means \pm standard deviation (SD). Statistical analysis was performed using SPSS 16.0 software (SPSS, Inc., Chicago, IL, USA). The Student's t-test was used for comparisons between 2 groups. For multiple-group comparisons, one-way analysis of variance (ANOVA) was performed. A value of $\mathrm{P}<0.05$ was considered to indicate a statistically significant difference.

\section{Results}

The expression of miR-429 is upregulated under hypoxic conditions. Firstly, the expression of miR-429 was evaluated. miR-429 expression was significantly increased after the cells were subjected to culture under hypoxic conditions compared with the control group (Fig. 1). This result suggests that the expression of miR-429 is upregulated under conditions mimicking myocardial ischemia.

miR-429 is effectively verexpressed and silenced. To examine the role of miR-429 in hypoxia-induced apoptosis, miR-429 was artificially overexpressed and silenced by transfection with miRNA-mimics and miRNA-inhibitor, respectively. The expression of miR-429 was significantly higher in the miRNA-

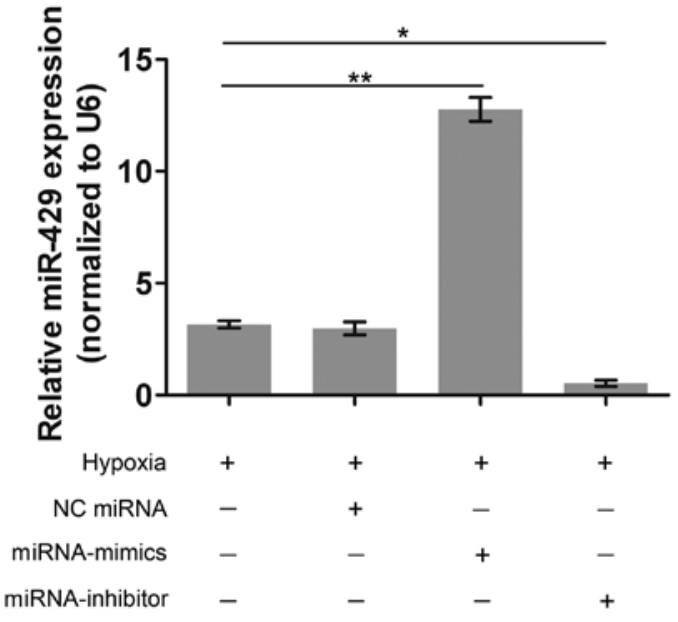

Figure 2. miR-429 was effectively overexpressed and silenced. miR-429 expression was markedly increased by transfection with miRNA-mimics and inhibited by transfection with miRNA-inhibitor. Each experiment was performed in triplicate; ${ }^{*} \mathrm{P}<0.05$ and ${ }^{* *} \mathrm{P}<0.01$ in comparison with the hypoxia group.

mimics group than that in the hypoxia group (Fig. 2). On the contrary, miR-429 expression was barely observed in the miRNA-inhibitor group. These results demonstrated that the transfection with miRNA-mimics and miRNA-inhibitor was effective in altering miR-429 expression in the AC-16 cells.
A

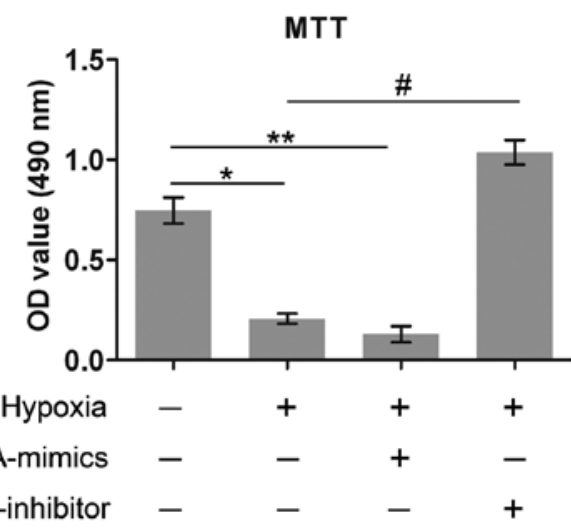

C

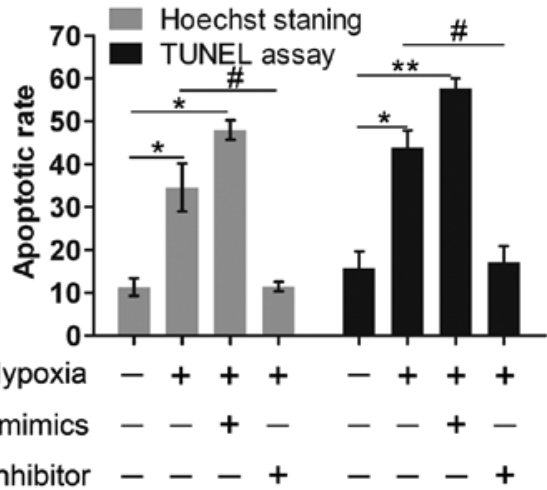

B

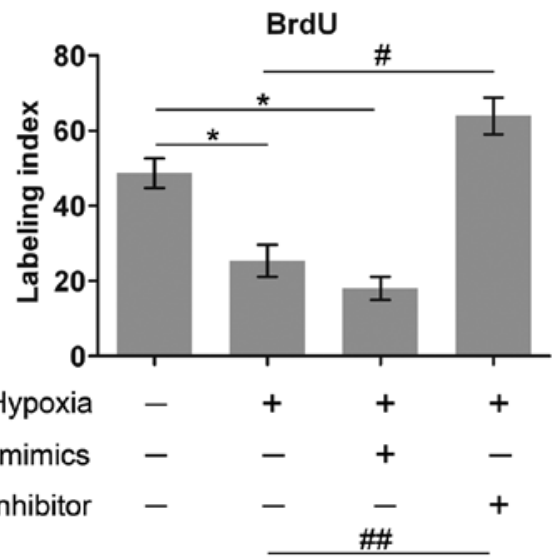

$\mathrm{D}$

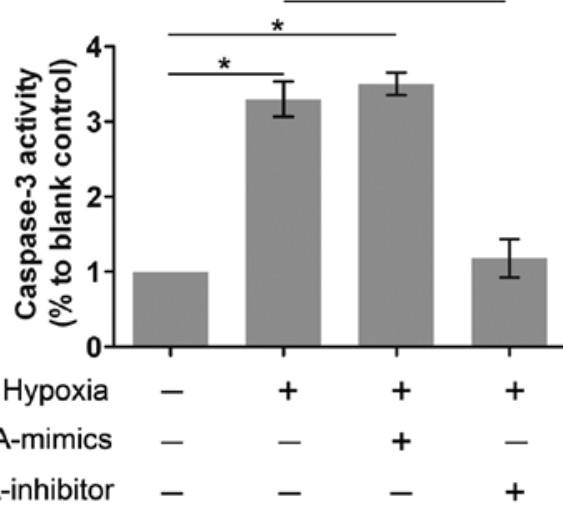

Figure 3. miR-429 affects cell viability, proliferation and apoptosis. MTT assay, BrdU assay, Hoechst staining, TUNEL assay and caspase-3 activity assay were performed. Culture under hypoxic conditions decreased cell viability and proliferation, but increased cell apoptosis. (A) Cell viability and (B) proliferation were inhibited by miR-429 overexpression, but these effects were reversed by the silencing of miR-429. (C) Cell apoptosis was significantly promoted by the overexpression of miR-429, and this effect was reversed by the silencing of miR-429. (D) Caspase-3 activity was increased by miR-429 overexpression, but decreased by the silencing of miR-429. Each experiment was performed in triplicate; ${ }^{~} \mathrm{P}<0.05$ and ${ }^{* *} \mathrm{P}<0.01$ in comparison with the control group; ${ }^{~} \mathrm{P}<0.05$ and ${ }^{\# \prime} \mathrm{P}<0.01$ in comparison with the hypoxia group. 
A

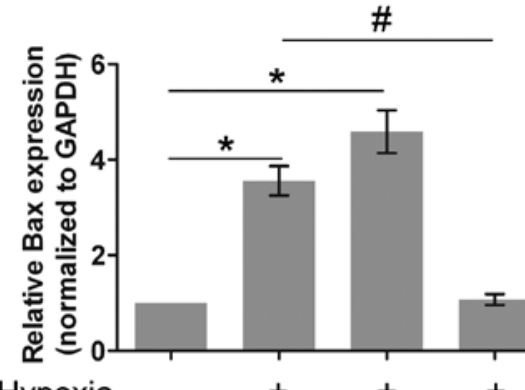

B

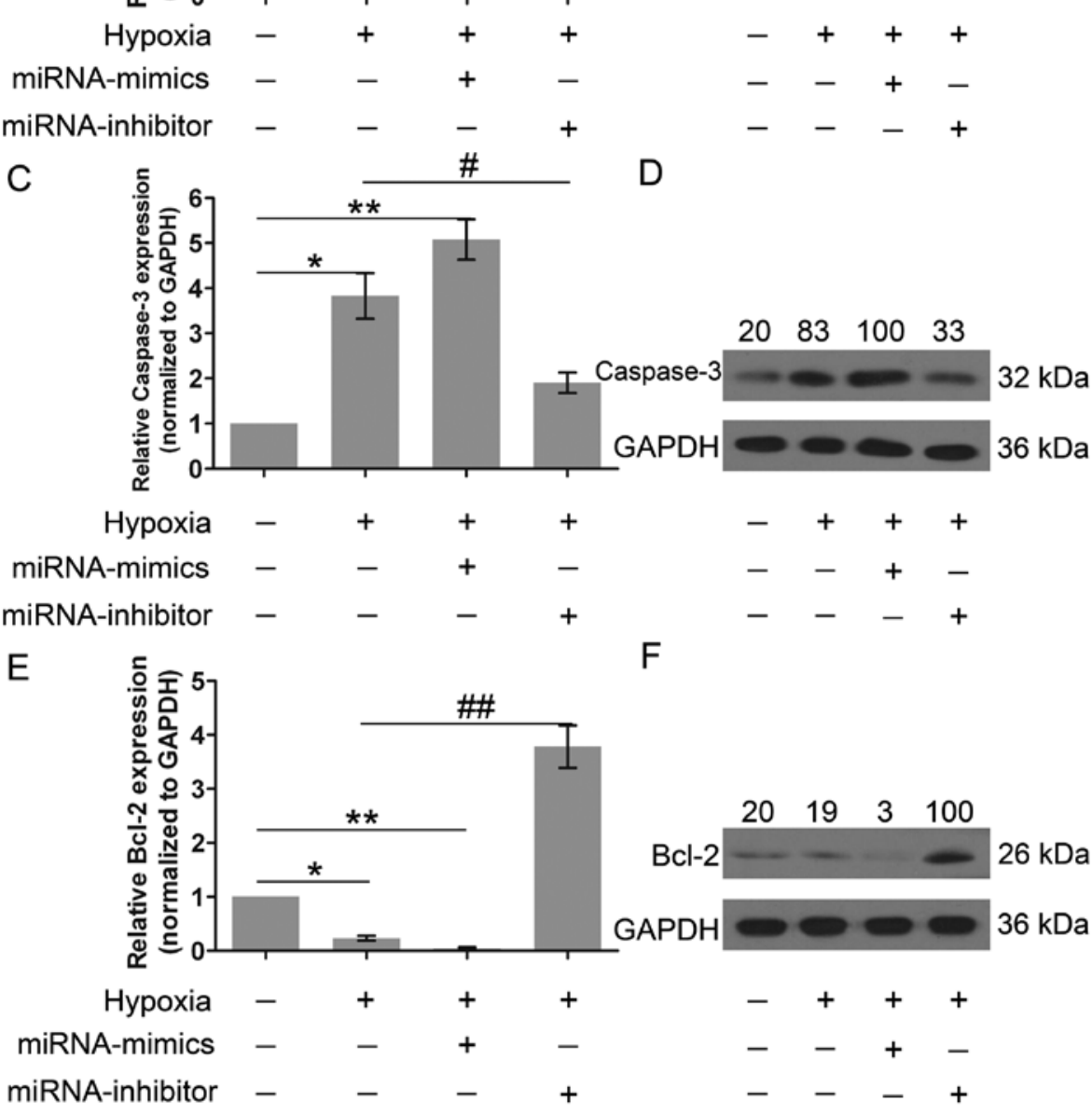

Figure 4. miR-429 plays a role in regulating the expression of Bax, caspase-3 and Bcl-2. Hypoxic culture and miR-429 overexpression increased the expression of Bax and caspase-3, but inhibited the expression of Bcl-2. The silencing of miR-429 had the opposite effects. (A and B) Bax expression. (C and D) Caspase-3 expression. (E and F) Bcl-2 expression. Each experiment was performed in triplicate; ${ }^{*} \mathrm{P}<0.05$ and ${ }^{* *} \mathrm{P}<0.01$ in comparison with the control group; ${ }^{*} \mathrm{P}<0.05$ and ${ }^{\# \#} \mathrm{P}<0.01$ in comparison with the hypoxia group.

Cell viability, proliferation and apoptosis are affected by miR-429 expression. We then examined the effects of miR-429 on cell viability, proliferation and apoptosis. Compared with the control group, the culture under hypoxic conditions and miR-429 overexpression markedly inhibited cell viability, whereas the knockdown of miR-429 expression significantly restored cell viability under hypoxic conditions, which was even higher than those of the control group (Fig. 3A). We hypothesized that miR-429 was associated with key genes involved in modulating cell viability, but the detailed mechanism remains to be investigated. Similar results were also recorded with the BrdU assay to evaluate cell proliferation (Fig. 3B). In addition, the results of Hoechst staining and TUNEL assay revealed that hypoxia markedly promoted cell apoptosis, and miR-429 overexpression also increased the apoptotic rate (Fig. 3C). By contrast, the silencing of miR-429 exerted potent protective effects against apoptosis; the apoptotic rate was markedly reduced following the silencing of miR-429. Caspase- 3 is an indicator of cell apoptosis. Exposure to hypoxia and the high expression of miR-429 markedly enhanced caspase-3 activity; however, the silencing of miR-429 exerted inhibitory effects on caspase-3 activity (Fig. 3D). On the whole, all these results indicated that hypoxia and the overexpression of miR-429 both impaired cell survival, whereas the knockdown of miR-429 expression greatly contributed to cell survival.

The expression of apoptosis-related genes is altered by the miR-429 expression. Subsequently, to further investigate the role of miR-429 in cell apoptosis, the expression of Bax, caspase- 3 and $\mathrm{Bcl}-2$ was explored. Compared with the control group, the level of expression of Bax and caspase- 3 was markedly increased following culture under hypoxic conditions 

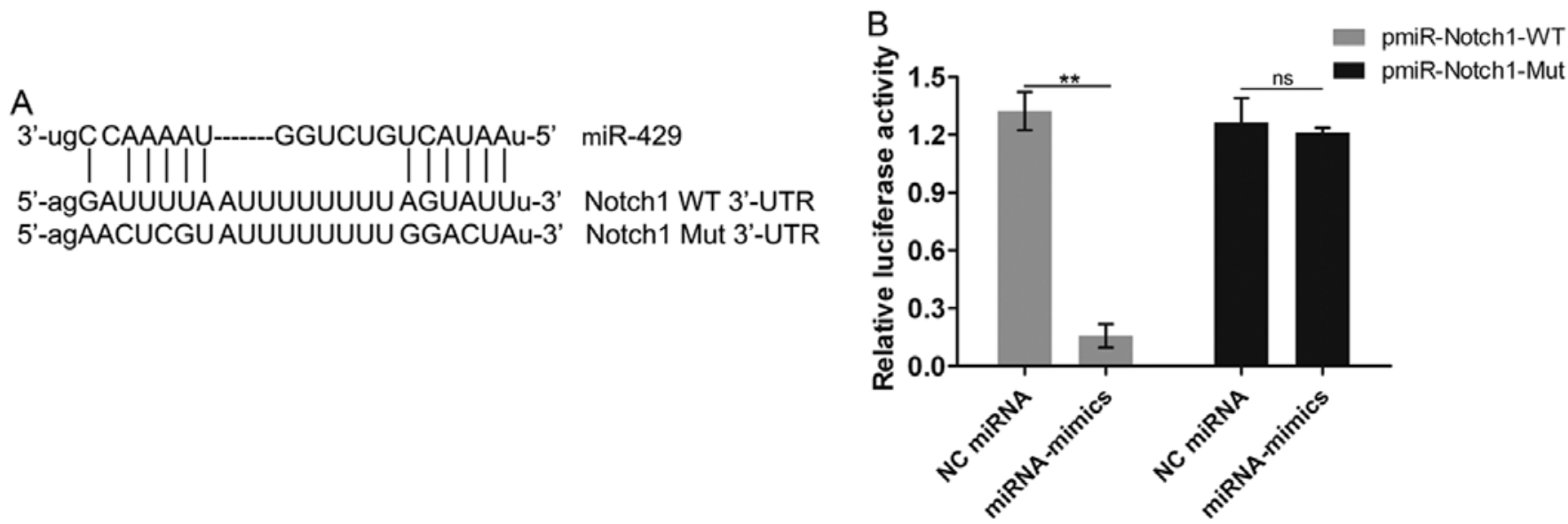

Figure 5. Notch1 is the target gene of miR-429. Dual-Luciferase Reporter assay was performed. (A) The binding sites with miR-429 in the 3'-untranslated regions (3'UTR) of Notch1 mRNA. (B) miR-429 overexpression inhibited the luciferase activity of WT Notch1, but not that of Mut Notch1. Each experiment was performed in triplicate; ${ }^{* *} \mathrm{P}<0.01$ in the comparison of the NC miRNA group vs. miRNA-mimics group; ns, no statistical significance.

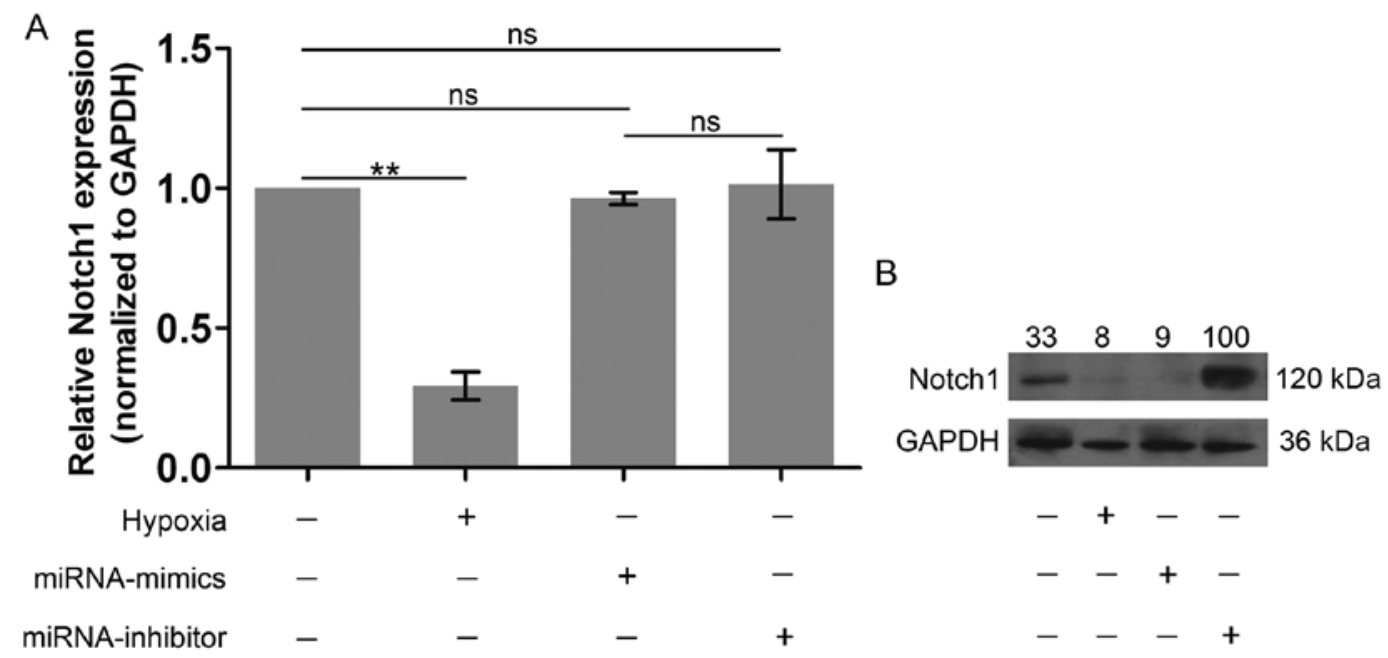

Figure 6. miR-429 overexpression suppresses the protein expression of Notch1. Culture under hypoxic conditions markedly decreased Notch1 expression. (A) Notch1 expression at the mRNA level was not markedly altered by the variations in miR-429 expression. (B) However, the overexpression of miR-429 inhibited Notch1 expression, whereas the silencing of miR-429 markedly promoted Notch1 expression, only at the protein level, as shown by western blot analysis. Each experiment was performed in triplicate; ${ }^{* *} \mathrm{P}<0.01$ in comparison with the blank control group; ns, no statistical significance.

and by miR-429 overexpression (Fig. 4A-D). However, Bax and caspase-3 were minimally expressed in the cells lacking miR-429 expression. Fig. 4E and F demonstrate that Bcl-2 was highly expressed in the miRNA-inhibitor group, indicating that miR-429 is a negative regulator of Bcl-2 expression. In short, these results suggest that miR-429 regulates cell apoptosis and survival.

Notchl is identified as the target gene of miR-429. We then aimed to explore the mechanisms underlying the regulatory effects of miR-429 on cell apoptosis. We predicted that Notch1 was a potential target gene of miR-429 through our analysis using the TargetScan, Pictar and microRNA. org databases. We then aimed to confirmed this prediction through Dual-Luciferase Reporter assay. miR-429 was shown to have some binding sites in the 3'-UTR of the WT Notch1 mRNA (Fig. 5A). Moreover, miR-429 overexpression markedly inhibited luciferase activity in the pmiR-Notch1-WT, not in the pmiR-Notch1-Mut (Fig. 5B), demonstrating that
miR-429 interacts with the Notch1 gene and possibly regulates the expression of Notch1; namely, Notch1 is the target gene of miR-429.

Notchl expression is inhibited by miR-429 overexpression, but is promoted by the silencing of miR-429 at the protein level. As we already confirmed that Notch1 was one of the target genes of miR-429, we further examined the role of miR-429 in regulating the expression of Notch1. Notch1 expression was notably suppressed following culture under hypoxic conditions (Fig. 6). It was noteworthy that the expression of Notch1 at the mRNA level was not significantly altered by miR- 429 overexpression or miR-429 silencing (Fig. 6A). In particular, in the protein level, Notch1 expression after the knockdown of miR-429 expression significantly exceeded its own expression in the hypoxia group, whereas miR-429 overexpression markedly inhibited Notch1 expression (Fig. 6B), indicating that the silencing of miR-429 promoted Notch1 expression. On the whole, these results further confirm that Notch1 is the target gene of miR-429. 


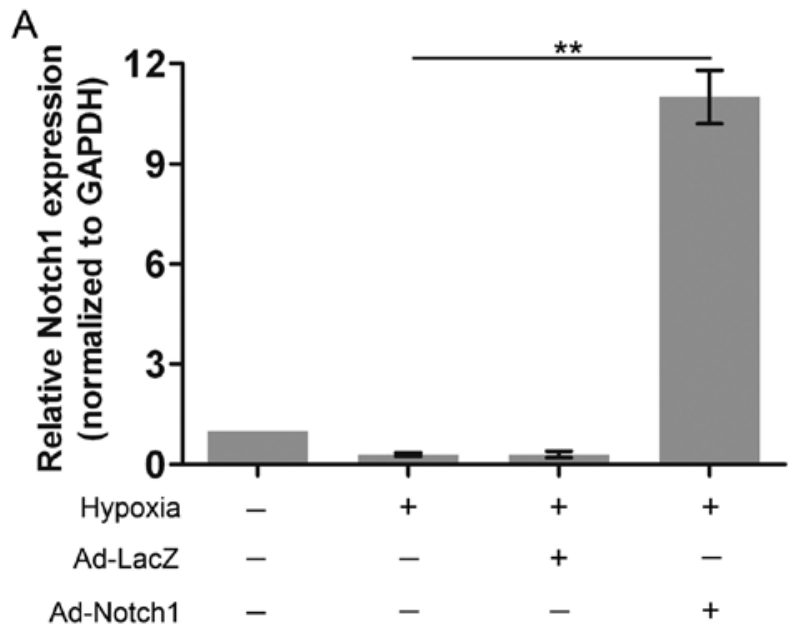

B

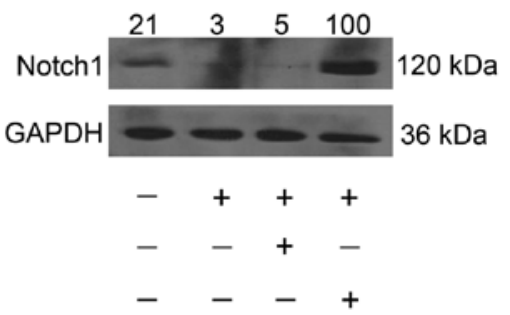

Figure 7. Notch1 is overexpressed by transfection with adenovirus. Notch1 expression was markedly increased following transfection with Ad-Notch1 compared with the hypoxia group at the (A) mRNA and (B) protein level. Each experiment was performed in triplicate; ${ }^{* *} \mathrm{P}<0.01$ in comparison with the hypoxia group.

A

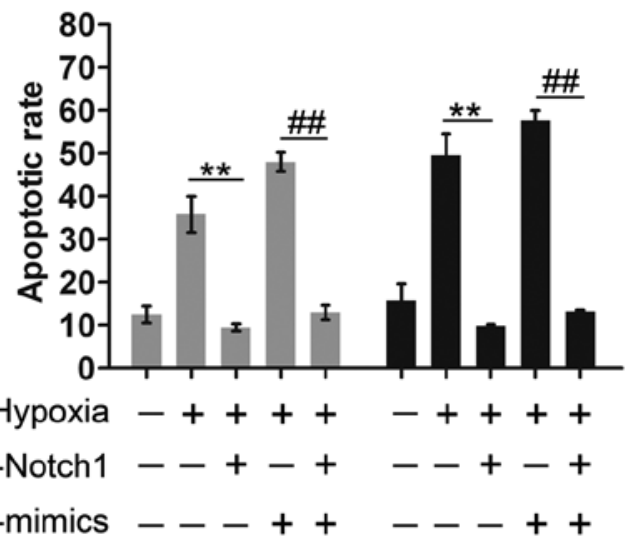

Hoechst staining

TUNEL assay

C
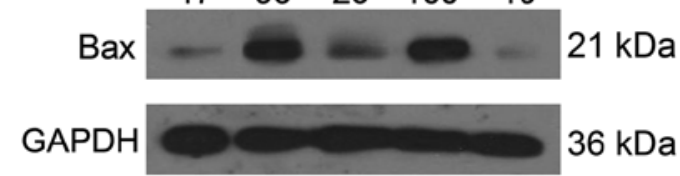

$\begin{array}{rlllll}\text { Hypoxia } & - & + & + & + & + \\ \text { Ad-Notch1 } & - & - & + & - & + \\ \text { miRNA-mimics } & - & - & - & + & +\end{array}$

B

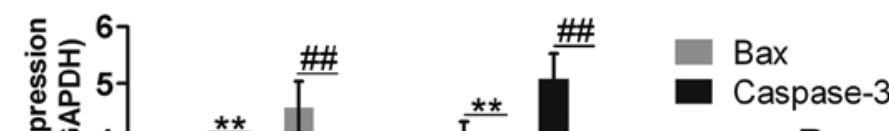

$\mathrm{D}$
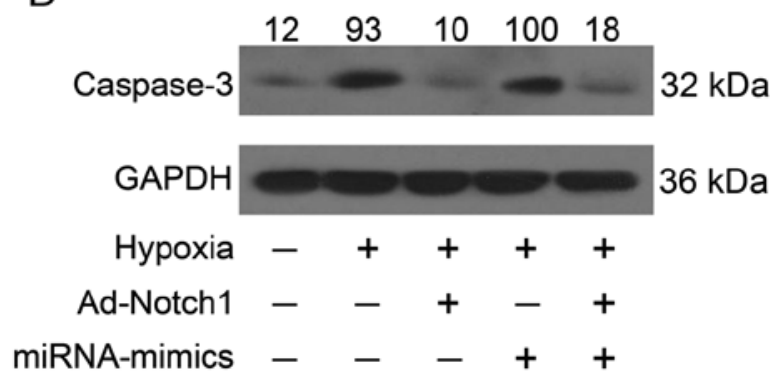

Figure 8. Notch1 overexpression diminishes cell apoptosis induced by hypoxia and reverses the effects of miR-429 overexpression. (A) Hoechst staining and TUNEL assay revealed that Notch1 overexpression significantly inhibited cell apoptosis. (B) Bax and caspase-3 expression were notably suppressed by the overexpression of the Notch1 at the mRNA level. The results of western blot analysis also confirmed the decreased expression of (C) Bax and (D) caspase-3 by the overexpression of the Notch1. Each experiment was performed in triplicate; ${ }^{* *} \mathrm{P}<0.01$ in comparison with the hypoxia group; ${ }^{\# \#} \mathrm{P}<0.01$ in comparison with the miRNA-mimics group.

Notch1 is overexpressed in AC-16 cells. Finally, to investigate whether miR-429 regulates hypoxia-induced apoptosis by regulating Notch1 expression, we overexpressed Notch1 to explore its role in cell apoptosis. Notch1 was markedly overexpressed at the mRNA level (Fig. 7A); moreover, its protein expression was also markedly increased compared with both the control group and the hypoxia group (Fig. 7B).
Notch1 overexpression reverses the effects of the miR-429 overexpression on cell apoptosis. Cell apoptosis was evaluated again following the co-overexpression of Notch1 and miR-429. The cells were co-transfected with miRNA-mimics and Ad-Notch1, harboring no specific binding sequences of the miR-429 in the 3'-UTR. Cell apoptosis was notably decreased by Notch 1 overexpression compared with the hypoxia 
group (Fig. 8A). Moreover, Notch1 overexpression inhibited cell apoptosis induced by miR-429 overexpression. In addition, Bax expression was markedly inhibited and caspase-3 expression was significantly reduced by Notch1 overexpression (Fig. 8B and C). Similarly, the increased expression of Bax and caspase- 3 induced by miR- 429 overexpression was reversed by the ectopic expression of Notch1. These results indicated that the high expression of Notch1 led to reduced apoptosis and provided cytoprotective effects against apoptosis, which also suggests that miR-429 regulates apoptosis by controlling Notch1 expression.

\section{Discussion}

In the present study, under hypoxic conditions mimicking myocardial ischemia, the expression of miR-429 was significantly upregulated, indicating that miR-429 is a characteristic molecule in myocardial ischemia. We then further explored the role of miR-429 in hypoxia-induced cell apoptosis. The results revealed that the miR-429-deprived cells were more resistant to hypoxia, implying that the downregulation of miR-429 provides cytoprotective effects against myocardial ischemia and hypoxia. In addition, Notch1 was also proven to be one of the target genes of miR-429 and to be involved in the anti-apoptotic effects. These findings may lead to the development of novel drugs to induce the downregulation of miR-429 and the upregulation of Notch1, which could possibly reduce myocardial ischemia disease and consequent MI and sudden death.

Cardiovascular disease is the leading cause of mortaliy worldwide (30). In particular, MI caused by coronary artery and myocardial ischemia remains one of the most challenging clinical concerns; myocardial ischemia plays a key role in the progression of cell apoptosis, cardiac damage and finally, heart failure (31). Heart-derived sudden death is also an issue which needs to be overcome. Traditionally, the rapid reperfusion of blood and oxygen is employed in the treatment of myocardial ischemia. However, rapid reperfusion often results in poor prognosis, characterized by cardiac injury and the apoptosis of cardiomyocytes. Other methods, including coronary artery bypass grafting and percutaneous coronary intervention, are effective in the majority of patients (32); however, the associated costs and secondary injuries are exorbitant. Hence, there is an urgent need for identifying novel therapeutic targets. Yang et al reported that radio-protective $105 \mathrm{kDa}$ protein (RP105) suppressed ischemia/reperfusion-induced myocardial injury by attenuating myocardial apoptosis (33). This finding inspired us to embark on a search to find an miRNA molecule that would regulate cell survival under hypoxic conditions. Some research into this matter has already been undertaken. Chen et al reported that in the $\mathrm{H}_{2} \mathrm{O}_{2}$-induced apoptosis of rat cardiomyocytes, the downregulation of miR-100 exerted cytoprotective effects (34). Another study by Tong et al indicated that the overexpression of miR-21 protected rat cardiomyocytes against apoptosis (35). These above-mentioned results demonstrate that the effects of miRNAs in cell apoptosis vary greatly. Compared with the results from the studies by Chen et al (34) and Tong et al (35), our results indicated for the first time, to the best of our knowledge, that the downregulation of miR-429 protects human cardiomyocytes under hypoxic conditions. miR-429 was naturally upregulated following culture under hypoxic conditions and was artificially silenced by transfection with miRNA-mimics. The silencing of miR-429 enhanced cell viability and proliferation, along with the decreased apoptotic rate and caspase-3 activity, implying that cell apoptosis was inhibited. Moreover, the decreased expression of Bax and caspase- 3 , and the increased expression of Bcl-2 confirmed the effects of miR-429 silencing. Besides, Notch1, as one of the target genes of miR-429, has been reported to contribute to the inhibition of the apoptosis of cardiomyocytes $(29,36)$. In this study, the overexpression of miR-429 significantly inhibited Notch1 expression. We consequently investigated the role of Notch1 in human cardiomyocytes. Its overexpression reversed the effects of miR-429 overexpression and reduced apoptosis, indicating that miR-429 regulates cell apoptosis possibly by regulating Notch1 expression.

In conclusion, our results demonstrate for the first time that the downregulation of miR-429 contributes to the survival of human cardiomyocytes, and inhibits apoptosis under hypoxic conditions. Notably, the overexpression of Notch1 significantly suppressed the apoptosis of human cardiomyocytes compared with the hypoxia group, thus providing a novel therapeutic target for the treatment of cardiac ischemia and the prevention of possible sudden death.

\section{References}

1. Jianqiang P, Ping Z, Xinmin F, Zhenhua Y, Ming Z and Ying G: Expression of hypoxia-inducible factor 1 alpha ameliorate myocardial ischemia in rat. Biochem Biophys Res Commun 465: 691-695, 2015.

2. Elsässer A, Suzuki K, Lorenz-Meyer S, Bode C and Schaper J: The role of apoptosis in myocardial ischemia: A critical appraisal. Basic Res Cardiol 96: 219-226, 2001.

3. Freude B, Masters TN, Robicsek F, Fokin A, Kostin S, Zimmermann R, Ullmann C, Lorenz-Meyer S and Schaper J: Apoptosis is initiated by myocardial ischemia and executed during reperfusion. J Mol Cell Cardiol 32: 197-208, 2000.

4. Kloner RA: Does reperfusion injury exist in humans? J Am Coll Cardiol 21: 537-545, 1993.

5. Elmore S: Apoptosis: A review of programmed cell death. Toxicol Pathol 35: 495-516, 2007.

6. Bracken CP, Whitelaw ML and Peet DJ: The hypoxia-inducible factors: Key transcriptional regulators of hypoxic responses. Cell Mol Life Sci 60: 1376-1393, 2003.

7. Choudhuri S: Small noncoding RNAs: Biogenesis, function, and emerging significance in toxicology. J Biochem Mol Toxicol 24: 195-216, 2010.

8. Fang J, Song XW, Tian J, Chen HY, Li DF, Wang JF, Ren AJ, Yuan WJ and Lin L: Overexpression of microRNA-378 attenuates ischemia-induced apoptosis by inhibiting caspase-3 expression in cardiac myocytes. Apoptosis 17: 410-423, 2012.

9. Hu S, Huang M, Li Z, Jia F, Ghosh Z, Lijkwan MA, Fasanaro P, Sun N, Wang X, Martelli F, et al: MicroRNA-210 as a novel therapy for treatment of ischemic heart disease. Circulation 122 (Suppl 11): S124-S131, 2010.

10. Qian L, Van Laake LW, Huang Y, Liu S, Wendland MF and Srivastava D: miR-24 inhibits apoptosis and represses Bim in mouse cardiomyocytes. J Exp Med 208: 549-560, 2011.

11. Li AY, Yang Q and Yang K: miR-133a mediates the hypoxiainduced apoptosis by inhibiting TAGLN2 expression in cardiac myocytes. Mol Cell Biochem 400: 173-181, 2015.

12. Wang X, Li C and Dai Q: Downregulation of microRNA-26b rescued hypoxia-induced apoptosis in cultured neonatal rat cardiac myocytes by regulating PTEN. Int J Clin Exp Med 8: 4073-4079, 2015.

13. Tang J, Li L, Huang W, Sui C, Yang Y, Lin X, Hou G, Chen X, Fu J, Yuan S, et al: MiR-429 increases the metastatic capability of HCC via regulating classic Wnt pathway rather than epithelialmesenchymal transition. Cancer Lett 364: 33-43, 2015.

14. Li J, Du L, Yang Y, Wang C, Liu H, Wang L, Zhang X, Li W, Zheng G and Dong Z: MiR-429 is an independent prognostic factor in colorectal cancer and exerts its anti-apoptotic function by targeting SOX2. Cancer Lett 329: 84-90, 2013. 
15. Gao H and Liu C: $\mathrm{miR}-429$ represses cell proliferation and induces apoptosis in HBV-related HCC. Biomed Pharmacother 68: 943-949, 2014.

16. Wang Y,Li M,Zang W, Ma Y, Wang N,Li P, Wang T and Zhao G: MiR-429 upregulation induces apoptosis and suppresses invasion by targeting Bcl-2 and SP-1 in esophageal carcinoma. Cell Oncol (Dordr) 36: 385-394, 2013.

17. Ye ZB, Ma G, Zhao YH, Xiao Y, Zhan Y, Jing C, Gao K, Liu ZH and Yu SJ: miR-429 inhibits migration and invasion of breast cancer cells in vitro. Int J Oncol 46: 531-538, 2015.

18. Lei W, Liu YE, Zheng Y and Qu L: MiR-429 inhibits oral squamous cell carcinoma growth by targeting ZEB1. Med Sci Monit 21: 383-389, 2015

19. von Boehmer H: Coming to grips with Notch. J Exp Med 194: F43-F46, 2001

20. Miele L and Osborne B: Arbiter of differentiation and death: Notch signaling meets apoptosis. J Cell Physiol 181: 393-409, 1999.

21. Li Y, Hiroi Y and Liao JK: Notch signaling as an important mediator of cardiac repair and regeneration after myocardial infarction. Trends Cardiovasc Med 20: 228-231, 2010.

22. Del Monte G, Grego-Bessa J, González-Rajal A, Bolós V and De La Pompa JL: Monitoring Notch1 activity in development: Evidence for a feedback regulatory loop. Dev Dyn 236 2594-2614, 2007.

23. Grego-Bessa J, Luna-Zurita L, del Monte G, Bolós V, Melgar P, Arandilla A, Garratt AN, Zang H, Mukouyama YS, Chen H, et al: Notch signaling is essential for ventricular chamber development. Dev Cell 12: 415-429, 2007.

24. Gude NA, Emmanuel G, Wu W, Cottage CT, Fischer K, Quijada P, Muraski JA, Alvarez R, Rubio M, Schaefer E and Sussman MA Activation of Notch-mediated protective signaling in the myocardium. Circ Res 102: 1025-1035, 2008.

25. Chiba S: Notch signaling in stem cell systems. Stem Cells 24 2437-2447, 2006.

26. del Monte G, Casanova JC, Guadix JA, MacGrogan D, Burch JB, Pérez-Pomares JM and de la Pompa JL: Differential Notch signaling in the epicardium is required for cardiac inflow development and coronary vessel morphogenesis. Circ Res 108 824-836, 2011.
27. Bolós V, Grego-Bessa J and de la Pompa JL: Notch signaling in development and cancer. Endocr Rev 28: 339-363, 2007.

28. Collesi C, Zentilin L, Sinagra G and Giacca M: Notch1 signaling stimulates proliferation of immature cardiomyocytes. J Cell Biol 183: 117-128, 2008.

29. Yu B and Song B: Notch 1 signalling inhibits cardiomyocyte apoptosis in ischaemic postconditioning. Heart Lung Circ 23: 152-158, 2014.

30. Gersh BJ, Sliwa K, Mayosi BM and Yusuf S: Novel therapeutic concepts: the epidemic of cardiovascular disease in the developing world: global implications. Eur Heart J 31: 642-648, 2010.

31. Eefting F, Rensing B, Wigman J, Pannekoek WJ, Liu WM, Cramer MJ, Lips DJ and Doevendans PA: Role of apoptosis in reperfusion injury. Cardiovasc Res 61: 414-426, 2004.

32. Velazquez EJ and Bonow RO: Revascularization in severe left ventricular dysfunction. J Am Coll Cardiol 65: 615-624, 2015.

33. Yang J, Guo X, Yang J, Ding JW,Li S, Yang R, Fan ZX and Yang CJ: RP105 protects against apoptosis in ischemia/reperfusion-induced myocardial damage in rats by suppressing TLR4-mediated signaling pathways. Cell Physiol Biochem 36: 2137-2148, 2015.

34. Chen A, Li G, Chen L, Guo J and Liu Y: Downregulation of microRNA-100 protects $\mathrm{H}_{2} \mathrm{O}_{2}$-induced apoptosis in neonatal cardiomyocytes. Int J Clin Exp Pathol 8: 5491-5496, 2015.

35. Tong Z, Jiang B, Wu Y, Liu Y, Li Y, Gao M, Jiang Y, Lv Q and Xiao X: MiR-21 protected cardiomyocytes against doxorubicininduced apoptosis by targeting BTG2. Int J Mol Sci 16: 14511-14525, 2015.

36. Boccalini G, Sassoli C, Formigli L, Bani D and Nistri S: Relaxin protects cardiac muscle cells from hypoxia/reoxygenation injury: Involvement of the Notch-1 pathway. FASEB J 29: 239-249, 2015. 